\title{
A Statistical Study on DH CMEs and Its Geoeffectiveness
}

\author{
V. Vasanth and S. Umapathy \\ School of Physics, Madurai Kamaraj University, Madurai 625021, India \\ Correspondence should be addressed to V. Vasanth; vasanth_velu2007@yahoo.co.in
}

Received 31 October 2013; Accepted 19 November 2013

Academic Editors: S. Koutchmy and Y.-D. Park

Copyright (C) 2013 V. Vasanth and S. Umapathy. This is an open access article distributed under the Creative Commons Attribution License, which permits unrestricted use, distribution, and reproduction in any medium, provided the original work is properly cited.

\begin{abstract}
A detailed investigation on geoeffectiveness of CMEs associated with DH-type-II bursts observed during 1997-2008 is presented. The collected sample events are divided into two groups based on their association with CMEs related to geomagnetic storms Dst $\leq-50 \mathrm{nT}$, namely, (i) geoeffective events and (ii) nongeoeffective events. We found that the geoeffective events have high starting frequency, low ending frequency, long duration, wider bandwidth, energetic flares, and CMEs than nongeoeffective events. The geoeffective events are found to have intense geomagnetic storm with mean Dst index $(-150 \mathrm{nT})$. There exists good correlation between the properties of CMEs and flares for geoeffective events, while no clear correlation exists for nongeoeffective events. There exists a weak correlation for geoeffective events between (i) CME speed and Dst index $(R=-0.51)$ and good correlation between (i) CME speed and solar wind speed $(R=0.60)$, (ii) Dst index and solar wind speed $(R=-0.64)$, and (iii) Dst index and southward magnetic field component $\left(B_{z}\right)(R=0.80)$. From our study we conclude that the intense and long duration southward magnetic field component $\left(B_{z}\right)$ and fast solar wind speed are responsible for geomagnetic storms, and the geomagnetic storms weakly depend on CME speed. About 22\% (50/230) of the DH-type-II bursts are associated with geomagnetic storms. Therefore the DH-type-II bursts associated with energetic flares and CMEs are good indicator of geomagnetic storms.
\end{abstract}

\section{Introduction}

CMEs are large scale plasma and magnetic field structure moving away from the sun into heliosphere. If the ejected CMEs appear to surround the occulting disk they are called halo CMEs [1]. The halo CMEs that appear on the visible disk are considered to be Earth-directed and cause disturbance to our Earth's magnetic field. The counterparts of CMEs in the interplanetary medium are called interplanetary CMEs (ICMEs), which are geoeffective because of the enhanced interplanetary magnetic field compressed by CME-driven shocks or by CMEs with strong magnetic field [2] and also due to the enhanced southward magnetic field component $\left(B_{z}\right)$ along the Sun-Earth direction in the interplanetary medium. The major geomagnetic storms are mainly caused by CMEs [3-5]. The geoeffectiveness of halo CMEs was established in earlier reports $[6,7]$ which showed that the halo CMEs are very much effective in producing geomagnetic storms. The statistical studies have found that the earth directed CMEs will reach earth within 1-5 days depending on the CME's initial speed $[8,9]$. Richardson et al. [10] investigated the geomagnetic storms with CMEs and concluded that the intense storms are produced by CMEs at any time in the solar cycle. The CMEs that are faster and wider are more energetic and can generate shocks in the interplanetary medium [11]. The type-II bursts are the earliest signature of shocks propagating outward in the solar corona. It is now well accepted that the DH-type-II bursts are produced by CME driven shocks $[9,12]$. Gopalswamy et al. [11] suggested that $\mathrm{DH}$-type-II bursts are associated with more energetic CMEs, and these CMEs are good indicator of geoeffectiveness if they appear on the front side of the solar disk. In the present paper we investigate the relationship between flares and CMEs associated with DH-type-II radio bursts and their role in causing geomagnetic storms. In Section 2 we will describe the method of data analysis, Section 3 will present the results, and the conclusion will be drawn in Section 4 .

\section{Data Analysis}

The data on DH-type-II bursts were obtained from Wind/WAVES type-II online catalog (http://cdaw.gsfc.nasa .gov/CME_list/radio/waves_type2.html) covering the period 
TABLE 1: Properties of type-II bursts, flares, and CMEs in geoeffective and nongeoeffective events.

\begin{tabular}{|c|c|c|c|c|}
\hline S. no. & Properties & Non-geoeffective events & Geoeffective events & $t$-test $P$ value (\%) \\
\hline & \multicolumn{4}{|c|}{ DH-type-II burst } \\
\hline 1 & Starting frequency $(\mathrm{MHz})$ & 10.14 & 10.99 & 21.32 \\
\hline 2 & Ending frequency $(\mathrm{MHz})$ & 2.38 & 1.52 & 7.09 \\
\hline 3 & Band width (MHz) & 7.76 & 9.46 & 2.45 \\
\hline \multirow[t]{2}{*}{4} & Duration (min) & 288 & 958 & 0.007 \\
\hline & \multicolumn{4}{|c|}{ Flares } \\
\hline 5 & Flare duration (min) & 50 & 70 & 6.3 \\
\hline 6 & Flare rise time $(\mathrm{min})$ & 25.97 & 35.18 & 6.9 \\
\hline \multirow[t]{2}{*}{7} & Flare decay time (min) & 24.66 & 35.12 & 10.55 \\
\hline & \multicolumn{4}{|c|}{ CMEs } \\
\hline 8 & CME speed $\left(\mathrm{km} \mathrm{s}^{-1}\right)$ & 1170 & 1263 & 35.39 \\
\hline 9 & Width (deg) & 247 & 337 & $\ll 1$ \\
\hline 10 & Acceleration $\left(\mathrm{m} \mathrm{s}^{-2}\right)$ & -4.25 & -19.23 & 5.05 \\
\hline
\end{tabular}

1997-2008 and containing 344 events. The DH-type-II bursts are recorded in the frequency range between $14 \mathrm{MHz}$ and $20 \mathrm{kHz}$ using RAD-2 (14-1 MHz) and RAD-1 (1 MHz$20 \mathrm{kHz}$ ) instruments by the Radio and Plasma Wave (WAVES) experiment on board the Wind spacecraft [13]. The data regarding flares associated with DH-type-II bursts (start time, end time, and peak time) are provided by the GOES soft X-ray flare catalog (http://www.ngdc.noaa.gov/stp/spaceweather/solar-data/solar-features/solar-flares/x-ray/goes). The data regarding the related CMEs (speed, width, and acceleration) are taken from the online SOHO/LASCO CME catalog [14] maintained by the CDAW data Center (http:// cdaw.gsfc.nasa.gov/CME_list). Out of 344 events only 230 events are taken for our further analysis using the following selection criteria.

(i) The given flare information should be clear (location and flare class should be reported).

(ii) The data gaps and Backside events are excluded from the analysis.

The above two selection criteria are used to select the clear events from which the properties of CMEs and their associated DH-type-II bursts can be studied clearly. The DHtype-II bursts associated with flares and CMEs are listed in Wind/WAVES type-II online catalog; we make use of the catalog. The temporal time window between DH-type-II bursts with flares and CMEs is $\pm 60 \mathrm{~min}$ form CME onset, but in some cases the time difference is longer; then the Type-II bursts start at very low frequency [15].

Recently Richardson and Cane [16] compiled a catalog of 340 ICMEs that passed Earth during 1996-2009. We utilized this ICME catalog for selecting the geoeffective events. We compared the CMEs listed in the ICME catalog [16] with 230 DH CMEs (DH-type-II bursts associated CMEs). The events are considered to be geoeffective if their Dst index $\leq-50 \mathrm{nT}$ [17]. A sample of $50 \mathrm{CMEs}$ in the ICME catalog coincides with $\mathrm{DH}$ CMEs (their Dst index values are $\leq-50 \mathrm{nT}$ ) and these 50 CMEs are termed geoeffective events. And the remaining 180 events are classified as nongeoeffective events.
Therefore the sample of 230 events (DH-type-II bursts associated with flares and CMEs) is compared with 340 ICMEs list [16]. The sample events are divided in to two groups as follows.

(i) Geoeffective events: It contains 50 events, whose DH CMEs are listed in the ICME list [16] and corresponding Dst index values $\leq-50 \mathrm{nT}$.

(ii) Nongeoeffective events: It contains the remaining 180 events, whose DH CMEs are not listed in ICME list [16].

\section{Results and Discussions}

The properties of Type-II bursts and their associated flares and CMEs in the geoeffective and nongeoeffective events were summarized in Table 1 . In Table 1, column 2 indicates the properties of Type-II bursts, flares, and CMEs, column 3 shows nongeoeffective, column 4 lists the geoeffective events, and column 5 represents the $t$-test $P$ value, the statistical significance of mean values of geoeffective, and nongeoeffective events ( $P \leq 5 \%$ means that there is $5 \%$ accidental difference is possible and the statistical significance is $95 \%)$.

3.1. Properties of Type-II Bursts. The distributions of starting and ending frequency of geoeffective (right) and nongeoeffective events (left) associated with DH-type-II bursts are presented in Figures 1(a)-1(d). The type-II burst starting frequency indicates the height at which shocks are being formed from the eruption [18]. The starting frequency of $\mathrm{DH}$ type-II bursts varies between 14 and $1 \mathrm{MHz}$, where $14 \mathrm{MHz}$ is the upper cutoff frequency of the WAVES instrument. Figures 1(a) and 1(b) show the distribution of starting frequency of DH-type-II bursts associated with nongeoeffective events and geoeffective events. About 46\% (82/180) of the nongeoeffective events and $54 \%(27 / 50)$ of the geoeffective events start at $14 \mathrm{MHz}$. The starting frequency of events at $14 \mathrm{MHz}$ implies that they cover the whole range of the WAVES instrument 


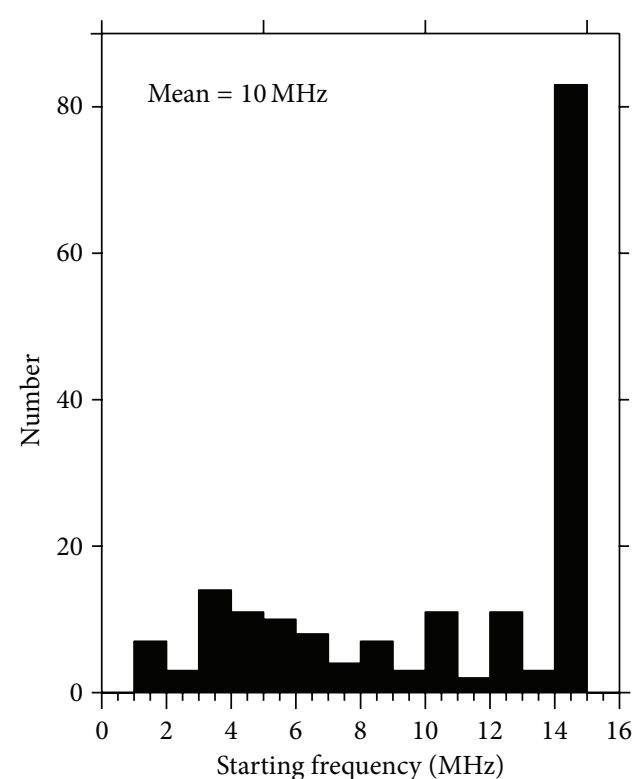

(a)

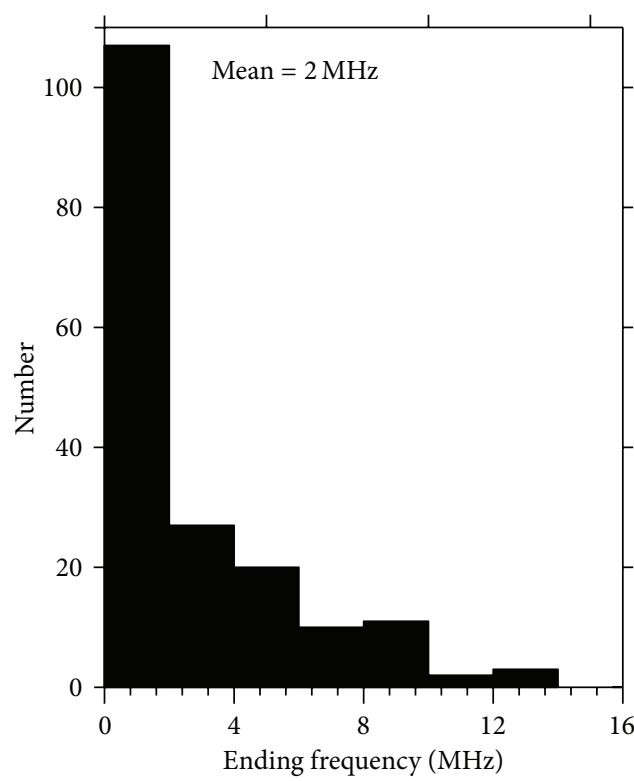

(c)

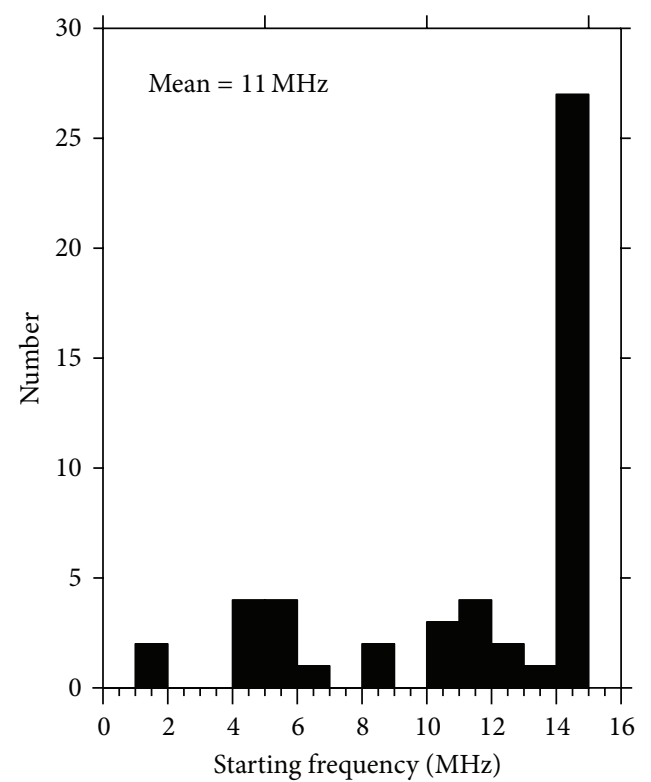

(b)

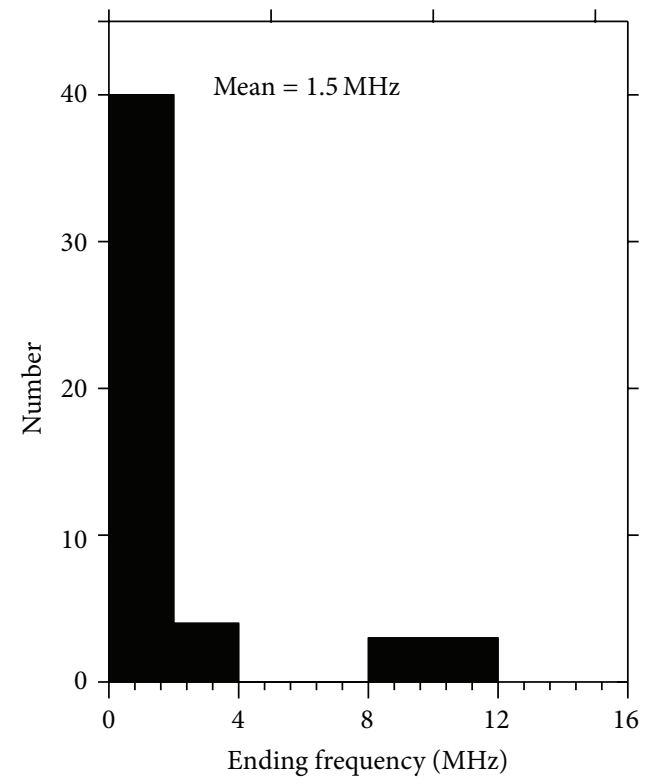

(d)

FIGURE 1: Distribution of starting frequency of DH-type-II bursts for (a) nongeoeffective eventsand (b) geoeffective events and distribution of ending frequency of DH-type-II bursts for (c) nongeoeffective events and (d) geoeffective events. In the Figure the geoeffective events are shown in right side and the nongeoeffective events on left side.

and some of them may be a continuation of metric type-II bursts. The mean starting frequency of geoeffective events is $11 \mathrm{MHz}$ and that of the nongeoeffective events is $10 \mathrm{MHz}$. The difference between the means is not statistically significant with $t$-test $P$ value $>5 \%$ as seen in Table 1 .

The type-II burst ending frequency indicates the energy of CMEs $[18,19]$; that is, when the kinetic energy of the associated CMEs are more then the shock can travel to larger distance in the interplanetary medium. The distributions of ending frequency of nongeoeffective events (left) and geoeffective events (right) are presented in Figures 1(c) and 1(d), respectively. The end frequency varies between $12 \mathrm{MHz}$ and $25 \mathrm{KHz}$. In nongeoeffective events 54\% (87/180) ends below $1 \mathrm{MHz}$, while in geoeffective events $76 \%(38 / 50)$ ends below $1 \mathrm{MHz}$. Most of the geoeffective events end around/below $1 \mathrm{MHz}$ implies that majority of the DH-type-II bursts continue to $\mathrm{km}$ wavelength range. The mean ending frequency of nongeoeffective events is $2 \mathrm{MHz}$ and that of the geoeffective events is $1.5 \mathrm{MHz}$. In both cases the ending frequency is below $2 \mathrm{MHz}$ which clearly indicates that the kinetic energy of the associated CMEs is larger, but they differ slightly in geoeffective and nongeoeffective events. The difference 


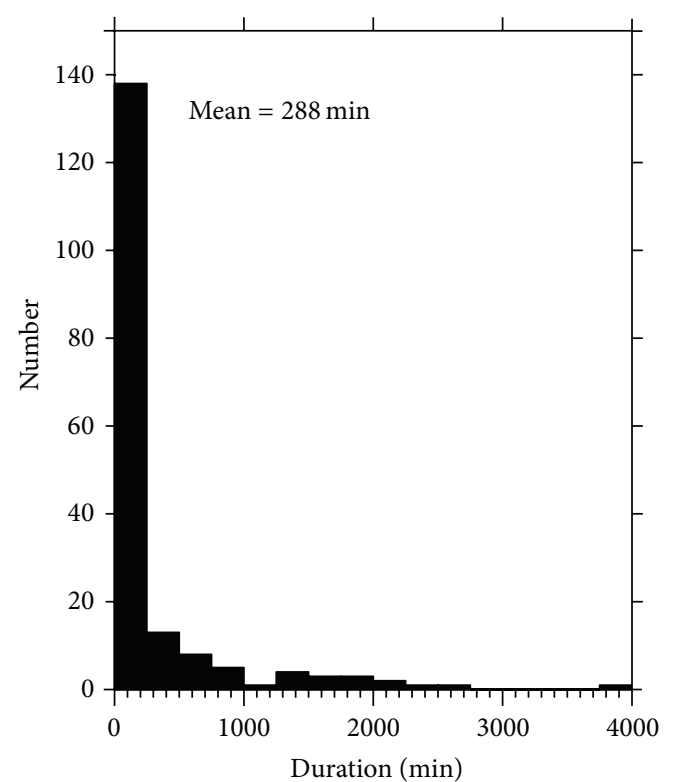

(a)

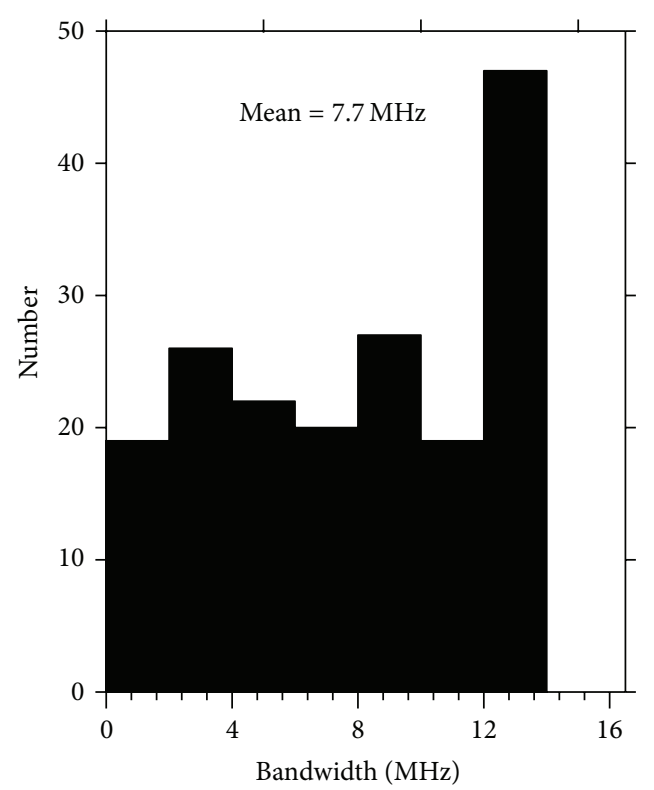

(c)

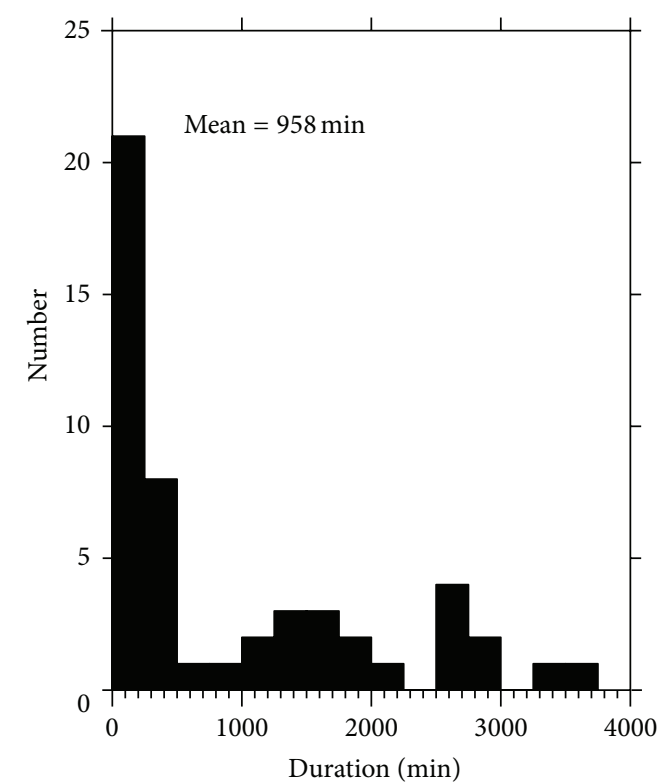

(b)

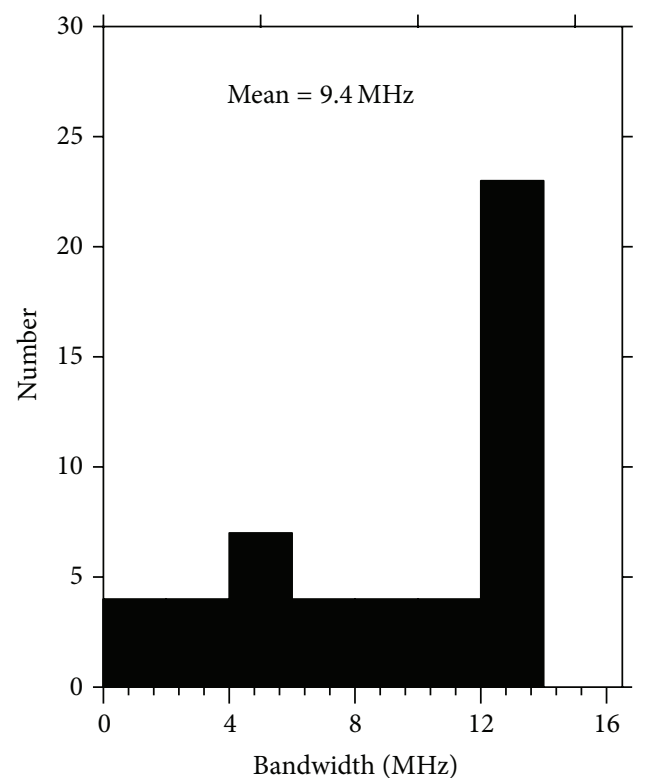

(d)

FIGURE 2: Distribution of duration of DH-type-II bursts for (a) nongeoeffective events and (b) geoeffective events and bandwidth of DHtype-II bursts for (c) nongeoeffective events and (d) geoeffective events. In the Figure the geoeffective events are shown in right side and the nongeoeffective events on left side.

between the means is less statistically significant with $t$-test $P$ value slightly greater than $5 \%$ as seen in Table 1 . Therefore the geoeffective events have highest starting frequency and lowest ending frequency compared to nongeoeffective events associated with DH-type-II bursts.

Figures 2(a) and 2(b) show the distribution of durations (the difference between the starting and ending time of the DH-type-II burst) for nongeoeffective (left) and geoeffective events (right), respectively. The duration of the DH-type-II bursts varies between few $\mathrm{min}$ and $4000 \mathrm{~min}$. In nongeoeffective events $76 \%(137 / 200)$ have duration within $200 \mathrm{~min}$ and the remaining $24 \%(43 / 180)$ have duration greater than 200 min; similarly for geoeffective events, $40 \%$ (20/50) have duration below $200 \mathrm{~min}$ and the remaining 60\% (30/50) have duration greater than $200 \mathrm{~min}$. The mean duration of the nongeoeffective events is $288 \mathrm{~min}$ and that of the geoeffective events is $958 \mathrm{~min}$. The duration of the DH-type-II bursts is longer for geoeffective events than nongeoeffective events. The difference between the means is statistically significant with $t$-test $P$ value $<1 \%$ as seen in Table 1 .

The distributions of bandwidths (the difference between starting and ending frequency of the DH-type-II burst) of 


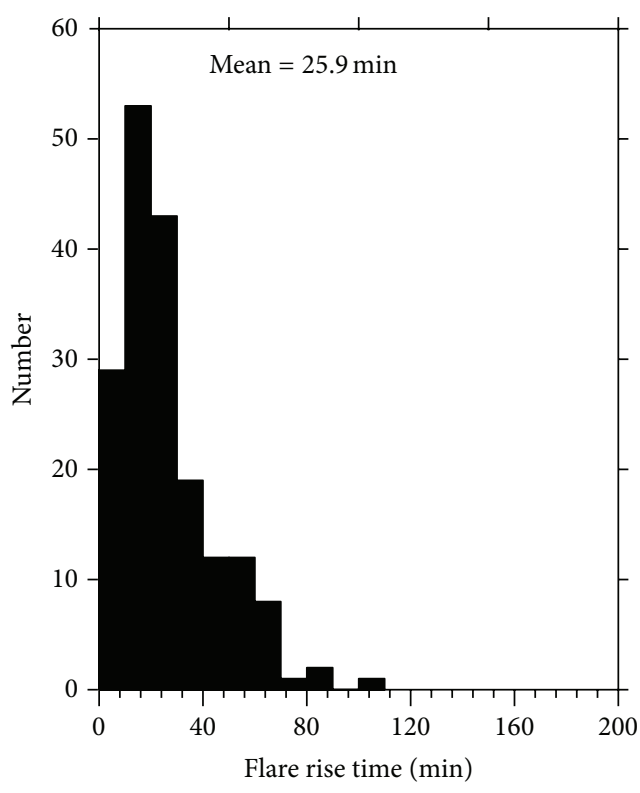

(a)

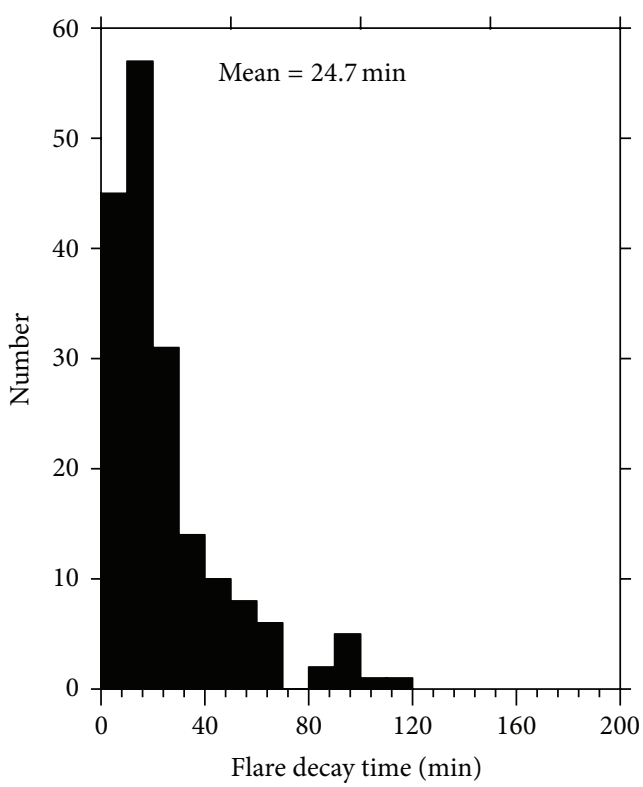

(c)

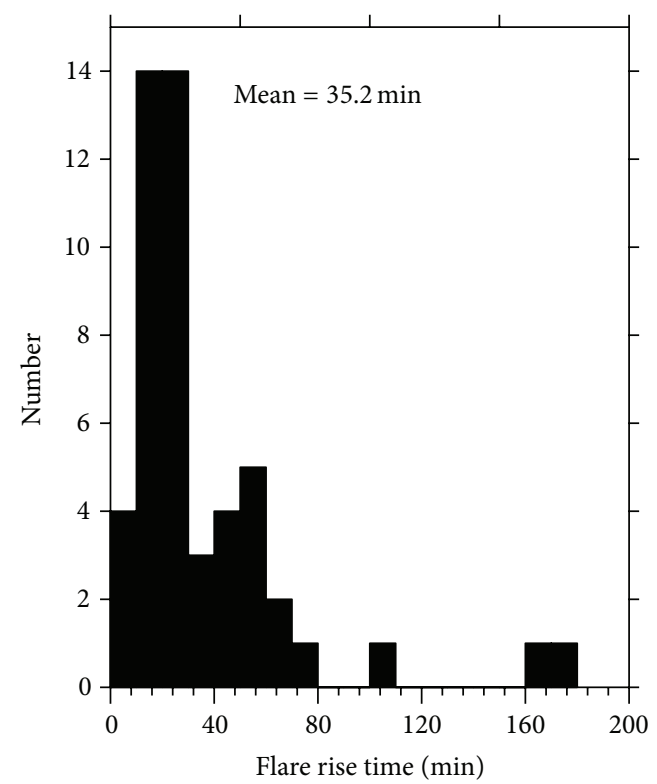

(b)

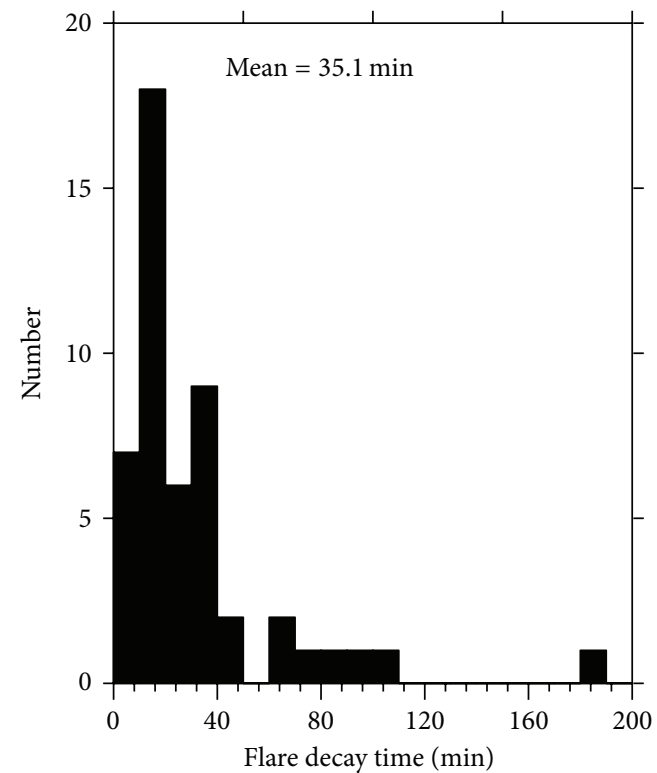

(d)

Figure 3: Distribution of flare rise time for (a) nongeoeffective events and (b) geoeffective events and flare decay time for (c) nongeoeffective events and (d) geoeffective events. In the Figure the geoeffective events are shown in right side and the nongeoeffective events on left side.

nongeoeffective events (left) and geoeffective events (right) are shown in Figures 2(c) and 2(d), respectively. Majority of the DH-type-II bursts in both groups have bandwidths less than $13 \mathrm{MHz}$. Only $15 \%$ of nongeoeffective events (27/180) have bandwidth greater than $13 \mathrm{MHz}$. On other hand, 38\% of geoeffective events (19/50) have bandwidth greater than $13 \mathrm{MHz}$. The mean bandwidth of nongeoeffective events is $8 \mathrm{MHz}$ and that of the geoeffective events is $9 \mathrm{MHz}$. The difference between the means is statistically significant with $t$-test $P$ value $<1 \%$ as seen in Table 1 .
3.2. Properties of Associated Flares. The distributions of flare rise times (the difference between flare starting time and flare peaking time) for nongeoeffective events (left) and geoeffective events (right) are shown in Figures 3(a) and 3(b), respectively. The flare rise times can be used to establish the relation between flares-type-II bursts [20]. The flare rise times vary between few min and $180 \mathrm{~min}$. In nongeoeffective events $72 \%$ of the flare peaks $(129 / 180)$ within $30 \mathrm{~min}$ of the flare start, while in geoeffective events $66 \%(33 / 50)$ peaks within $30 \mathrm{~min}$. The distribution decreases exponentially after the rise 
time in nongeoeffective events, while it is not a case for geoeffective events. The mean flare rise time of nongeoeffective events is $26 \mathrm{~min}$ and that of the geoeffective events is $35 \mathrm{~min}$. Vršnak et al. [21] reported that the acceleration of CMEs showed a fundamental relationship between the dynamic of CMEs and flare energy release. The majority of the DH-typeII bursts are produced after the flare rise times; therefore they decelerate, while the DH-type-II bursts that occur before the flare rise times are found to accelerate. The difference between the means is less statistically significant with $t$-test $P$ value slightly greater than $5 \%$ as seen in Table 1 .

Figures 3(c) and 3(d) show the distribution of flare decay times (the difference between flare peaking and flare ending time) for nongeoeffective events (left) and geoeffective events (right), respectively. The decay times of the flare varies between few $\mathrm{min}$ and $180 \mathrm{~min}$. In nongeoeffective events $75 \%(135 / 180)$ of the flares decay within $30 \mathrm{~min}$, while in geoeffective events $62 \%$ (31/50) of the flares decay within $30 \mathrm{~min}$. The mean decay times of nongeoeffective events is $25 \mathrm{~min}$ and that of the geoeffective events is $35 \mathrm{~min}$. The difference between the means is less statistically significant with $t$-test $P$ value slightly greater than $5 \%$ as seen in Table 1 .

The distributions of flare durations (the difference between flare starting time and flare ending time) for nongeoeffective (left) and geoeffective events (right) are shown in Figures 4(a) and 4(b), respectively. In nongeoeffective events $61 \%(110 / 180)$ have duration within $50 \mathrm{~min}$, while in geoeffective events 54\% (27/50) have duration within $50 \mathrm{~min}$. The mean flare duration of non-geoeffective events is $50 \mathrm{~min}$ and that of the geoeffective events is $70 \mathrm{~min}$. The flare duration of geoeffective events is found to be longer than that of nongeoeffective events. The longer flare duration or rise time is an implication of enhanced action of Lorentz force which indicates that the associated CME may have high speed and acceleration [22]. The difference between the means is less statistically significant with $t$-test $P$ value slightly greater than $5 \%$ as seen in Table 1 .

The distributions of flare classes associated with CMEs producing DH-type-II bursts for nongeoeffective (left) and geoeffective events (right) are shown in Figures 4(c) and 4(d), respectively. The $\mathrm{X}$-ray flare class is classified as $A, B, C, M$, and $X$ depending on their intensities: where $\left(A=10^{-8}\right.$, $B=10^{-7}, C=10^{-6}, M=10^{-5}, X=10^{-4} \mathrm{~W} \mathrm{~m}^{-2}$ ), the value after the flare class is the multiplication factor. For example, $X 5.4$ flare has a intensity value of $5.4 \times 10^{-4} \mathrm{~W} \mathrm{~m}^{-2}$. In nongeoeffective events $23 \%(41 / 180)$ are $X$-Class flares, $53 \%(96 / 180)$ are $M$-Class flares, $21 \%$ (37/180) are C-Class flares, and $3 \%(6 / 180)$ are $B$-Class flares, while in geoeffective events $40 \%(20 / 50)$ are $X$-Class flares, 38\% (19/50) are $M$ Class flares, and the remaining 22\% (11/50) are C-Class flares. The geoeffective events are associated with more energetic $X$-Class flares than nongeoeffective events. The association rate of flare class increases with flux of the flares [23, 24]. The CMEs associated with stronger flares will have high CME speed [25-27]. About $68 \%$ of the geoeffective events (36/50) and $60 \%$ of the nongeoeffective events (108/180) originate on the western hemisphere. The western source regions are better candidate for CMEs to be geoeffective [28].
3.3. Properties of Associated CMEs. The distributions of CME speed for nongeoeffective (left) and geoeffective events (right) are shown in Figures 5(a) and 5(b), respectively. The CME speed listed in the LASCO CME catalog is measured from the height time measurements projected in the sky plane. So all the measured parameters will suffer from projection effects; no attempt had been made to correct the projection effects. The speed of the CMEs varies from few hundred $\mathrm{km} \mathrm{s}^{-1}$ to $3000 \mathrm{~km} \mathrm{~s}^{-1}$. The mean speed of nongeoeffective events is $1170 \mathrm{~km} \mathrm{~s}^{-1}$ and that of the geoeffective events is $1263 \mathrm{~km} \mathrm{~s}^{-1}$. The difference between the means is not statistically significant with $t$-test $P$ value $>5 \%$ as seen in Table 1.

The distributions of apparent width of nongeoeffective (left) and geoeffective events (right) are presented in Figures 5(c) and 5(d), respectively. The angular width is the angular extent between the two edge position angles of the CME in the sky plane. The width of the CMEs varies between $30^{\circ}$ and $360^{\circ}$. The mean width of the nongeoeffective events is $247^{\circ}$ and that of the geoeffective events is $337^{\circ}$. The difference between the means is statistically significant with $t$-test $P$ value $<1 \%$ as seen in Table 1 . In geoeffective events $88 \%$ $(44 / 50)$ are halo CMEs, while in nongeoeffective events only $46 \%(83 / 180)$ are halo CMEs. Therefore the geoeffective events are found to be wider than nongeoeffective events.

The distributions of CME acceleration for nongeoeffective (left) and geoeffective events (right) are presented in Figures 5(e) and 5(f), respectively. We considered the CMEs with data point $\geq 4$, so that the acceleration is reliable. From the distributions it is clear that in both the geoeffective and nongeoeffective events tend to extend on decelerating side shows the tendency of CMEs to decelerate, while few nongeoeffective events show acceleration. The CMEs will accelerate/decelerate in the LASCO FOV depending on the CMEs interaction with the solar wind. The fast CMEs tend to decelerate, while the slow CMEs accelerate [14]. The mean acceleration of nongeoeffective events is $-4.25 \mathrm{~m} \mathrm{~s}^{-2}$ and that of the geoeffective events is $-19.23 \mathrm{~m} \mathrm{~s}^{-2}$. The difference between the means is less statistically significant with $t$-test $P$ value slightly greater than $5 \%$ as seen in Table 1 .

The correlations between CME speed and CME acceleration for nongeoeffective (left) and geoeffective events (right) associated with DH-type-II bursts are shown in Figures 6(a) and 6(b), respectively. There exists a good correlation between CME speed and acceleration for geoeffective events with correlation coefficient of $R=-0.78$, while no clear correlation exists for nongeoeffective events. Our result is similar to Gopalswamy et al. [11], who reported the properties of CMEs in limb and all events associated with DH-typeII bursts. Majority of the CMEs considered by Gopalswamy et al. [11] are decelerating events; therefore the acceleration is anticorrelated with the CME speed, which shows that the fast CMEs decelerate more. From Figure 6(b), it is clear that the acceleration decreases with increase in speed of CMEs; that is, the faster CMEs decelerate more than slower CMEs. Gopalswamy et al. [18] concluded that the faster CMEs decelerate more because of the drag force faced by the CMEs as they propagate in the interplanetary medium. 


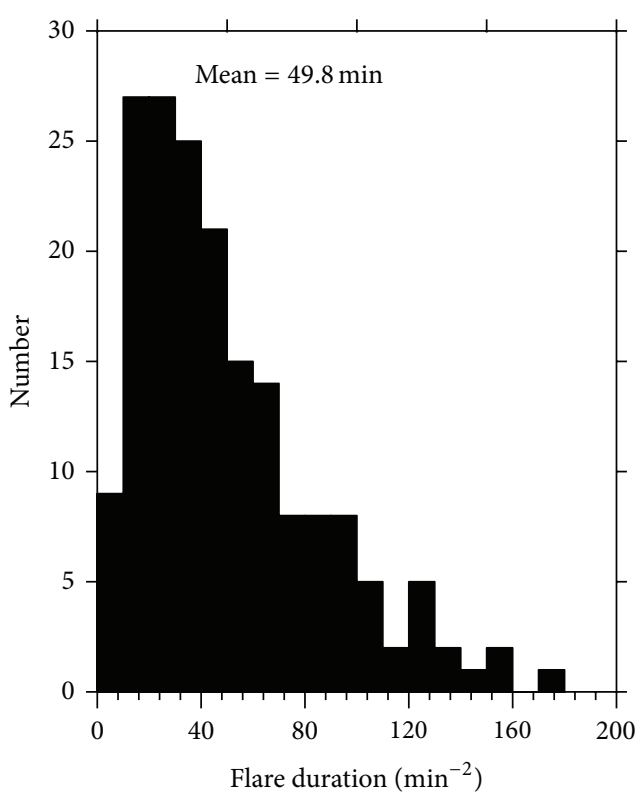

(a)

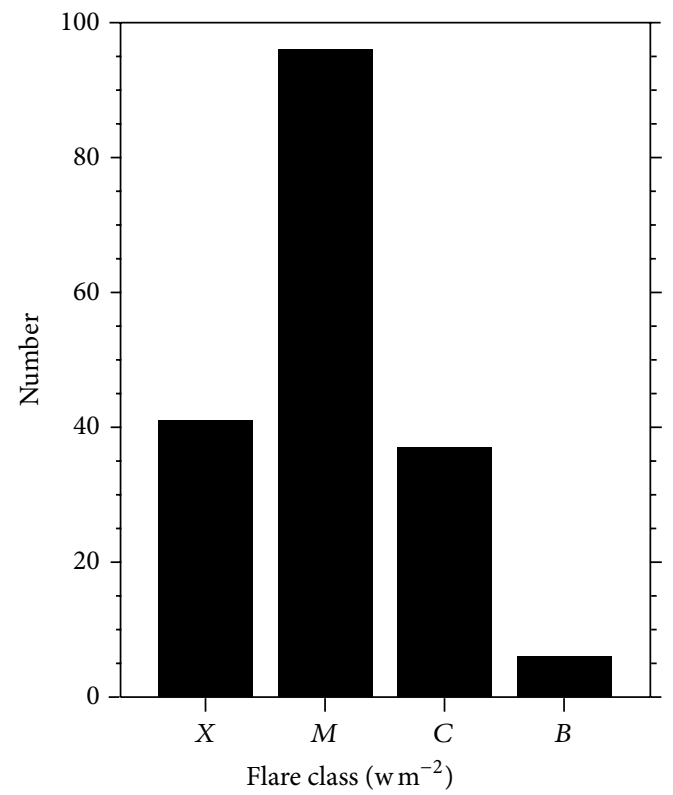

(c)

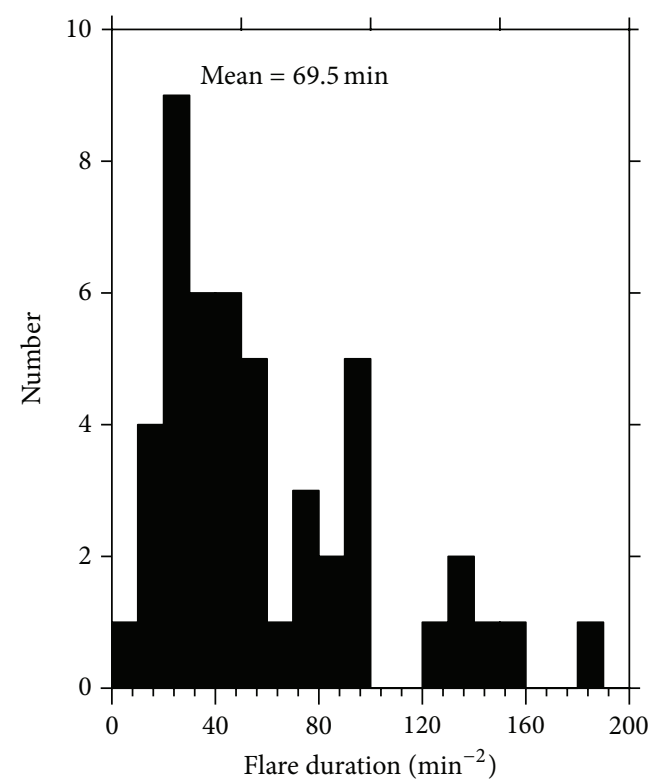

(b)

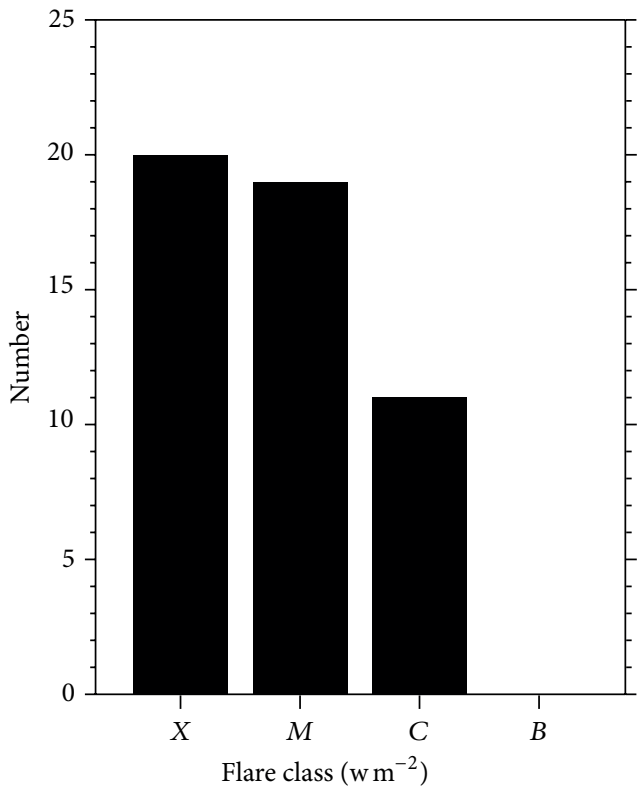

(d)

FIGURE 4: Distribution of flare duration for (a) nongeoeffective events and (b) geoeffective events and flare class for (c) nongeoeffective events and (d) geoeffective events. In the Figure the geoeffective events are shown in right side and the nongeoeffective events on left side.

The stronger flare associated CMEs are highly decelerated in LASCO FOV [26]. The relationship between CME speed and CME acceleration were studied by several authors [11, 14, $21,29]$ in which acceleration increases with speed, while in our result the CME decelerates (acceleration decreases) with increase in CME speed and the anticorrelation is due to the aerodynamical drag [24, 30-33]. The action of Lorentz force plays an important role in acceleration/deceleration of CMEs. Longer action of Lorentz force is found in accelerating CMEs, while it acts shorter in decelerating CMEs. As a result the drag force dominates the dynamic of CMEs with decrease in
Lorentz and gravity force, and the CMEs decelerate in the LASCO FOV [21]. The anticorrelation between CME speed and acceleration is consistent with the earlier reports [14, 21, 33]. Yashiro et al. [14] reported that CME speed $(>900 \mathrm{~km} / \mathrm{s})$ tends to decelerate in the LASCO FOV.

The arrival time of CMEs at earth can be predicted using empirical models $[34,35]$. The CME speed changes in interaction with the solar wind speed. The arrival time model uses the CME speed as the primary input and estimates the travel time of CMEs at earth. The fast CMEs decelerating in the LASCO FOV will arrive with shorter travel time than 


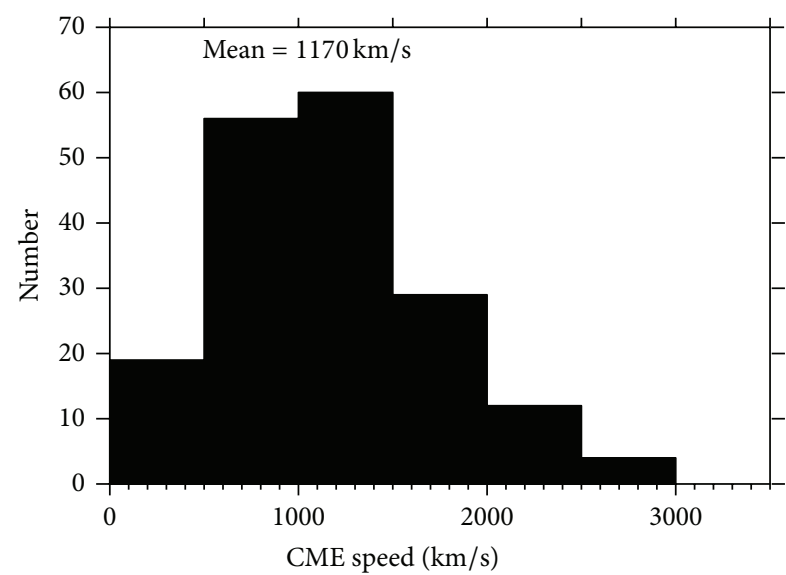

(a)

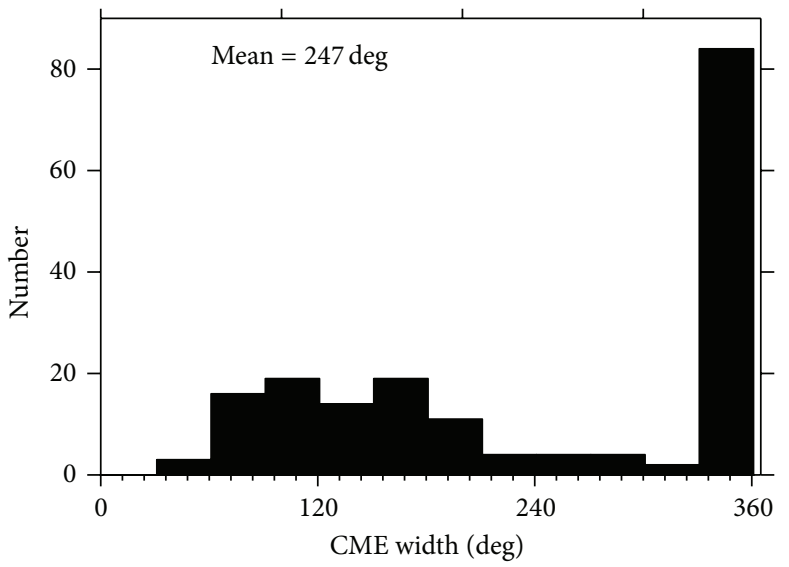

(c)

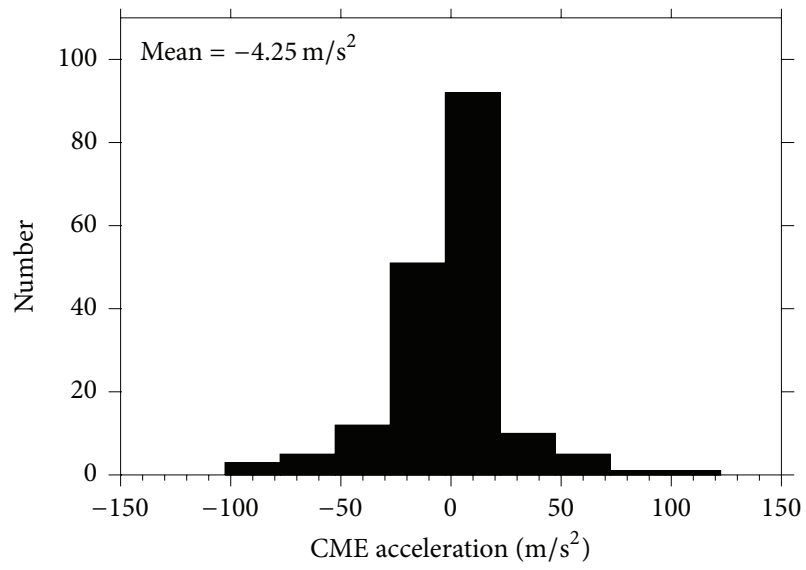

(e)

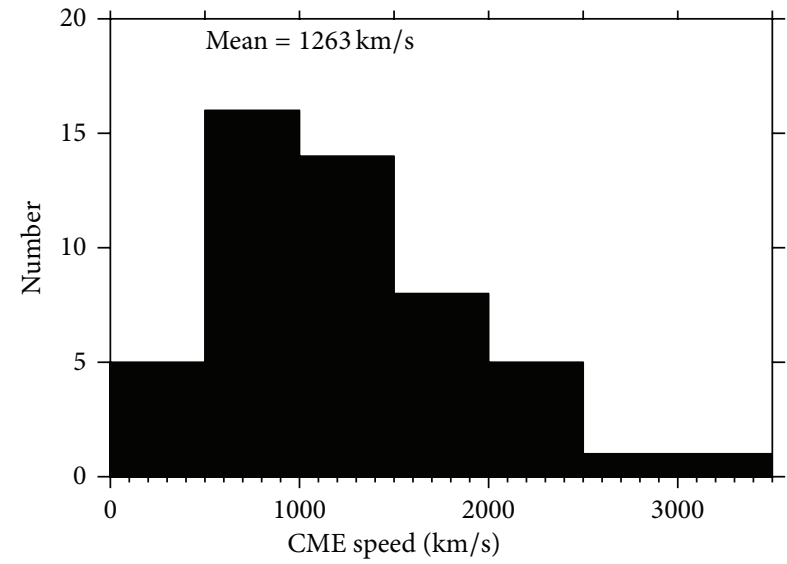

(b)

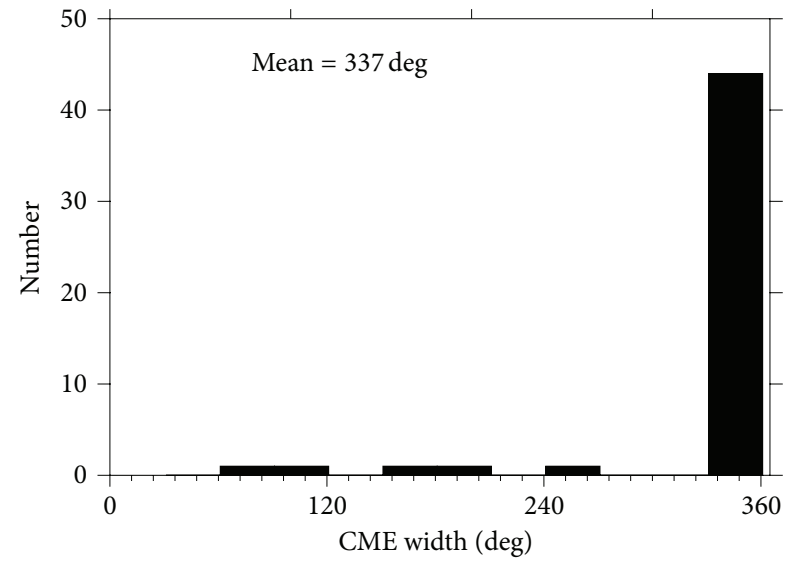

(d)

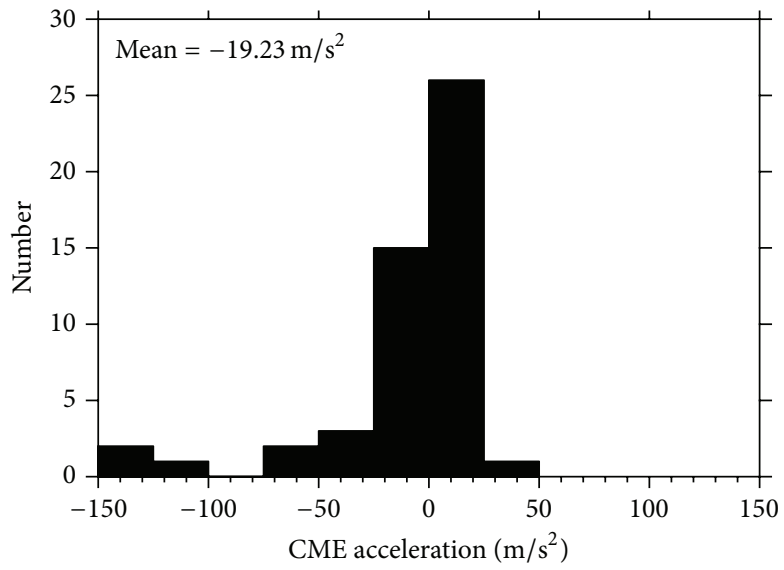

(f)

FIGURE 5: Distribution of speed of CMEs for (a) nongeoeffective events and (b) geoeffective events, width of CMEs for (c) nongeoeffective events and (d) geoeffective events, and acceleration of CMEs for (e) nongeoeffective events and (f) geoeffective events. In the Figure the geoeffective events are shown in right side and the nongeoeffective events on left side.

slow CMEs, which have longer travel time. The arrival time predicted by model also contains error, since the CMEs observed and listed by LASCO CME catalog suffer from projection effects. If the projection effects are corrected then the error in estimating the arrival time also decreases.
The correlations between CME speed and flare flux for nongeoeffective (left) and geoeffective events (right) are shown in Figures 7(a) and 7(b), respectively. The value of flare flux is obtained from the X-ray flare class; the value after the flare class is the multiplication factor. For example, X5.4 


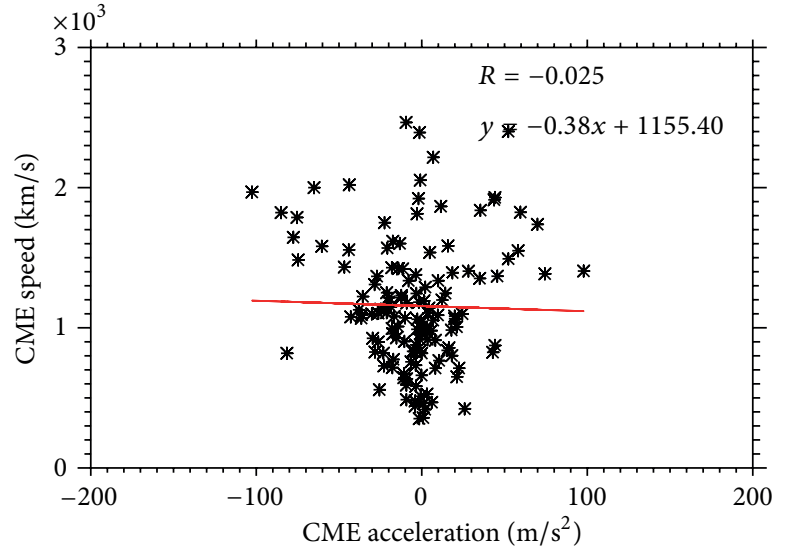

(a)

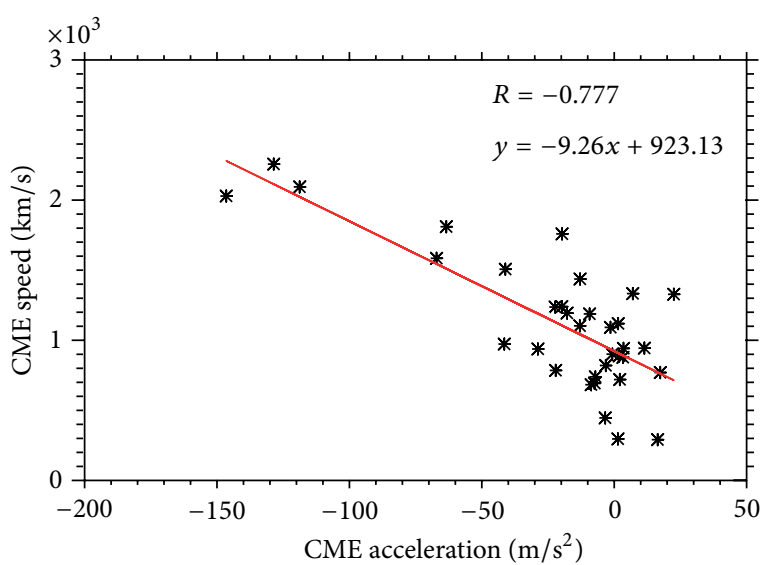

(b)

Figure 6: Correlation between CMEs speed and acceleration of CMEs for (a) nongeoeffective events and (b) geoeffective events. In the Figure the geoeffective events are shown in right side and the nongeoeffective events on left side.

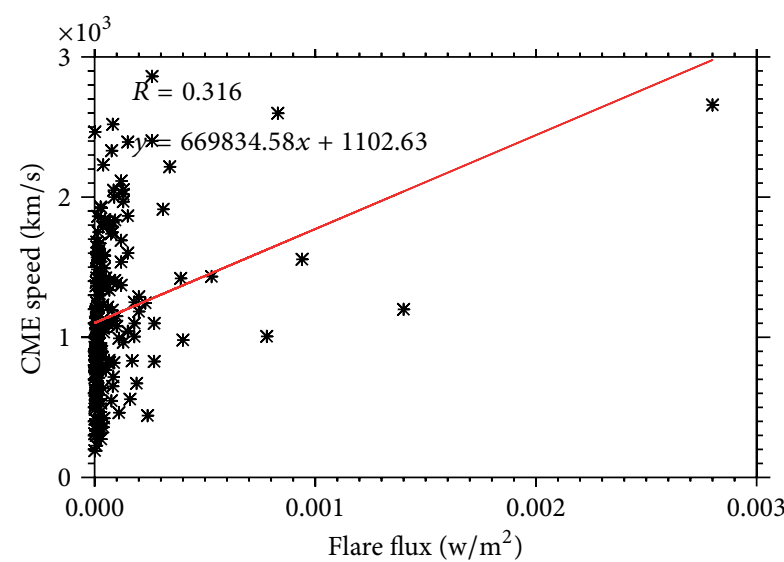

(a)

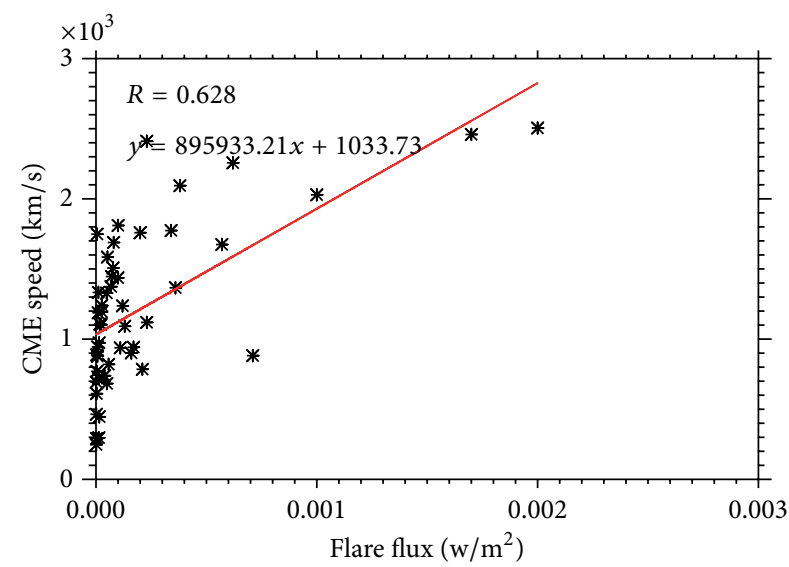

(b)

FIGURE 7: Correlation between CMEs speed and flare flux for (a) nongeoeffective events and (b) geoeffective events. In the Figure the geoeffective events are shown in right side and the nongeoeffective events on left side.

has a flux of $5.4 \times 10^{-4} \mathrm{~W} \mathrm{~m}^{-2}$. The correlation is used to investigate the flare-CME relationship. The relation between strength of the flare and CME speed was studied by several authors [36-40]. We found a similar relation between the two parameters. There exists a weak correlation between CME speed and flare flux for nongeoeffective events $(R=0.32)$ and good correlation for geoeffective events $(R=0.63)$. The correlation shows that the faster CMEs are associated with more energetic flares. Earlier report by Moon et al. [26] found a correlation coefficient of $R=0.47$ between flare flux and CME speed, while Yashiro and Gopalswamy [41] reported a better correlation coefficient of $R=0.56$. The stronger flares are associated with faster CMEs in the geoeffective events, while the weak correlation in the nongeoeffective events is due to the lack of stronger flares.

The correlations between CME acceleration and flare flux for nongeoeffective (left) and geoeffective events (right) are shown in Figures 8(a) and 8(b), respectively. There exists a good correlation between flare flux and CME acceleration for geoeffective events with a correlation coefficient of $R=$ -0.80 , while no clear correlation exists for nongeoeffective events. The CMEs associated with stronger flares are highly decelerated due to dominant action of drag force. Maričić et al. [39] obtained a good positive correlation between CME peak acceleration and flare flux during the acceleration phase.

The disturbance in the earth magnetic field caused by the impact of interplanetary magnetic field with enhanced southward magnetic field component is termed as Geomagnetic storms. The average change in horizontal component of earth's magnetic field measured in $\mathrm{nT}$ at low latitude is known as disturbance storm time (Dst). The minimum Dst index value is the intensity of the storm. The geomagnetic storms are classified as weak $(-30 \mathrm{nT} \leq$ Dst $\leq-50 \mathrm{nT})$, moderate $(-50 \mathrm{nT} \leq$ Dst $\leq-100 \mathrm{nT})$, intense $(-100 \mathrm{nT} \leq$ Dst $<-200 \mathrm{nT})$, severe $(-200 \mathrm{nT} \leq \mathrm{Dst} \leq-350 \mathrm{nT})$, and super storm (Dst $<-350 \mathrm{nT}$ ) [42]. The CMEs with Dst $\leq$ $-50 \mathrm{nT}$ are considered to be geoeffective [17]. The distribution of Dst index and correlation between CME speed and Dst 


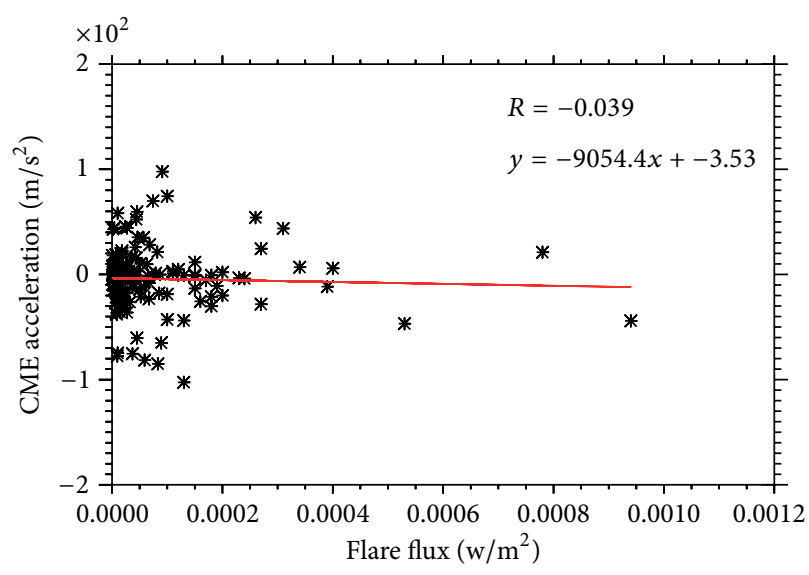

(a)

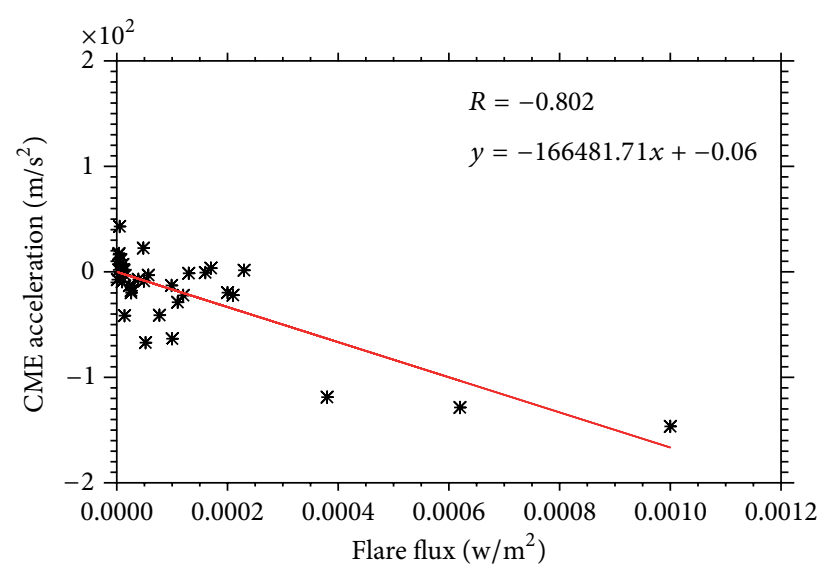

(b)

FIGURE 8: Correlation between CMEs acceleration and flare flux for (a) nongeoeffective events and (b) geoeffective events. In the Figure the geoeffective events are shown in right side and the nongeoeffective events on left side.

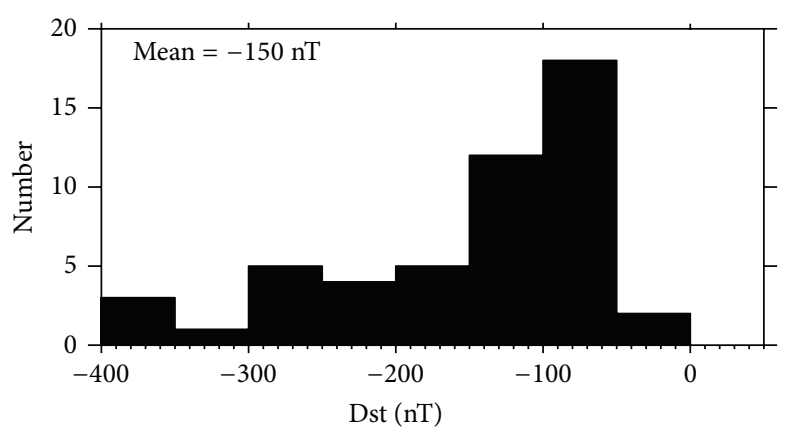

(a)

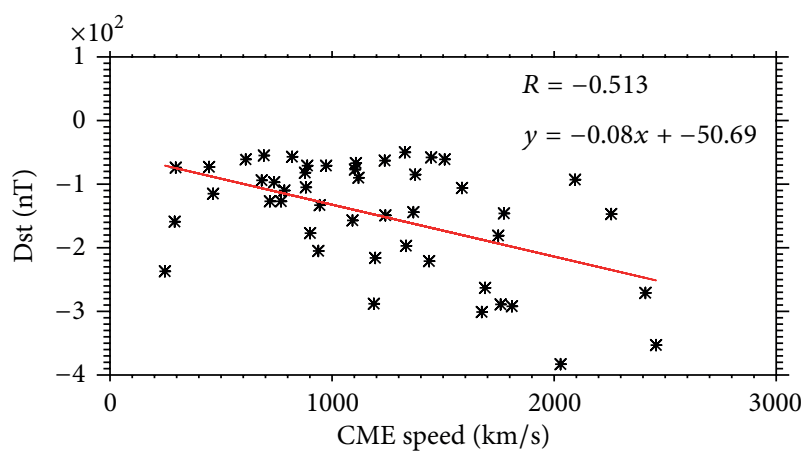

(b)

FIGURE 9: (a) Distribution of Dst index for geoeffective events and (b) correlation between CMEs speed and Dst index for geoeffective events.

index for geoeffective events are presented in Figures 9(a) and $9(\mathrm{~b})$, respectively. In Figure $9(\mathrm{a})$ the Dst value varies between $-50 \mathrm{nT}$ and $-400 \mathrm{nT}$ with mean Dst index (-150 nT). Therefore the mean value is found to be intense geoeffective.

There exists a weak correlation between CME speed and Dst index with a correlation coefficient of $R=-0.51$ as shown in Figure 9(b); the anticorrelation indicates that the Dst value decreases with increase in CME speed. Our result is similar to that of Gopalswamy et al. [43]. They investigated the dependence of geoeffectiveness with CME speed and they obtained a weak correlation with correlation coefficient of $R=-0.46$. The weak correlation indicates that the geoeffectiveness weakly depends on CME speed.

There exists a good correlation between Dst index and southward magnetic field component $\left(B_{z}\right)$ with a correlation coefficient of $R=0.80$ as shown in Figure 10(a). The very less scattering in the correlation plot shows that there exists a relationship between Dst index and $B_{z}$; the geoeffectiveness strongly depends on $B_{z}$ values. The intense $B_{z}$ corresponds to intense geomagnetic storm. Therefore the south magnetic field component plays an important role in the generation of geomagnetic storms.
The correlation between solar wind speed and Dst index for geoeffective events are shown in Figure 10(b); there exists a good anticorrelation between Dst index and solar wind speed with correlation coefficient of $R=-0.64$, excluding the two outliers. The less scattering in the correlation plot shows that there exists a relationship between them and the geoeffectiveness depends on solar wind speed in the interplanetary medium. The fast solar winds are found to be associated with intense geomagnetic storm.

The correlation between CME speed and solar wind speed for geoeffective events are shown in Figure 11(a). There exists a good correlation with a correlation coefficient of $R=0.60$, excluding the two outlier points. The correlation shows that there is a relationship between the CME speed and solar wind speed. Therefore the CME speed in the interplanetary medium is affected by the solar wind speed. The fast CMEs are found to have more solar wind speed.

The correlation between CME speed and $B_{z}$ are shown in Figure 11(b). No correlation exists between them.

Therefore it is concluded that the geoeffectiveness of the CMEs strongly depend on interplanetary parameters such as solar wind speed and $B_{z}$ and weakly depend on CME speed. 


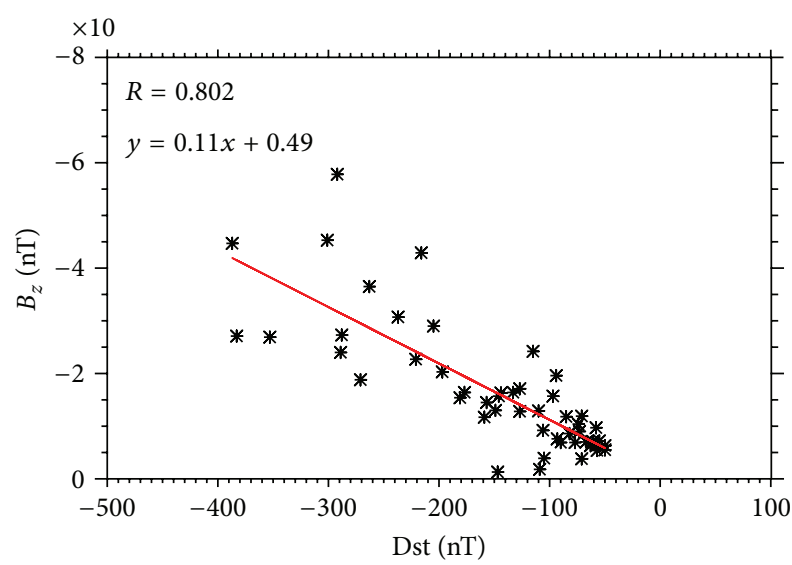

(a)

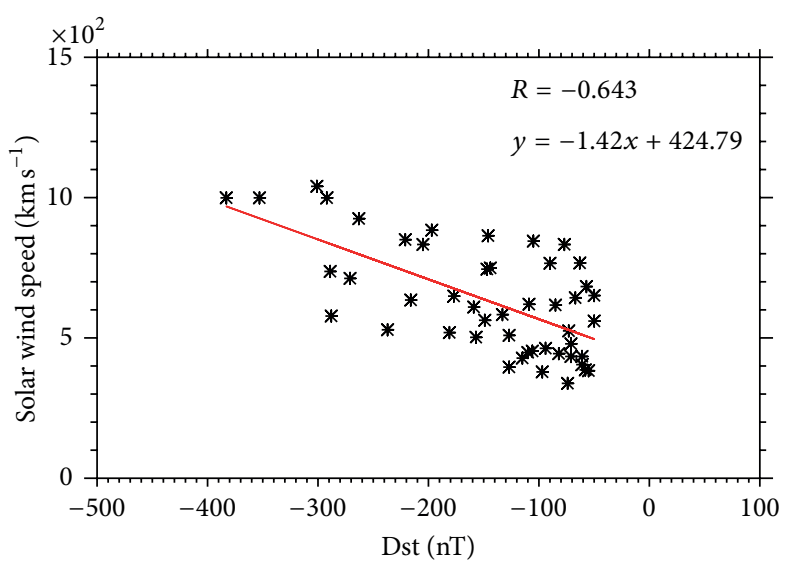

(b)

FIGURE 10: Correlation between (a) Dst index and southward magnetic field component $\left(B_{z}\right)$ and (b) Dst index and solar wind speed for geoeffective events.

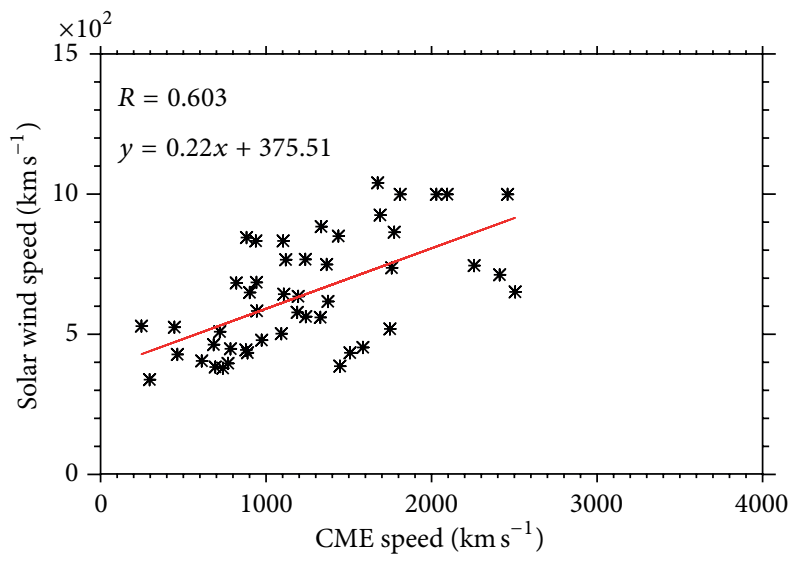

(a)

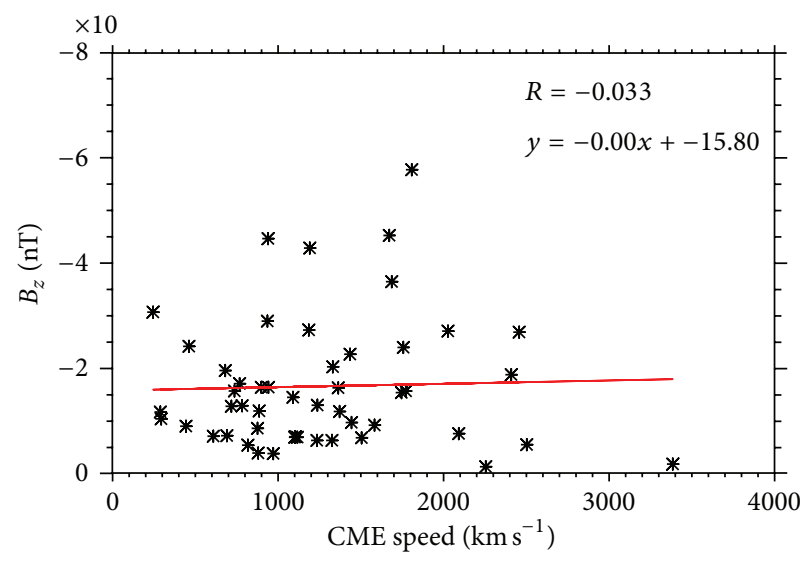

(b)

FIGURE 11: Correlation between (a) CME speed and solar wind speed and (b) CME speed and southward magnetic field component $\left(B_{z}\right)$ for geoeffective events.

The main cause for the arrival of geomagnetic storms at earth is intense and long duration $B_{z}$ and solar wind speed.

\section{Conclusion}

We have analyzed 230 CMEs associated with flares and DHtype-II bursts observed during 1997-2008. The sample events are divided into two groups based on the CMEs association with geomagnetic storm Dst $\leq-50 \mathrm{nT}$ listed in the ICME catalog [16] as (i) geoeffective events and (ii) nongeoeffective events. The results of our study are summarized as follows.

(i) The DH-type-II bursts associated with geoeffective events are found to have high starting frequency, low ending frequency, and long duration compared to the DH-type-II bursts associated with nongeoeffective events.

(ii) The geoeffective events are associated with more energetic $X$-Class flares than nongeoeffective events. (iii) The geoeffective events are found to have faster and wider CMEs than the nongeoeffective events.

(iv) There exists a good correlation between CME speed and acceleration for geoeffective events with correlation coefficient of $R=-0.78$, while no clear correlation exists for nongeoeffective events.

(v) There exists a good correlation between flare flux and CME acceleration for geoeffective events with a correlation coefficient of $R=-0.80$, while no clear correlation exists for nongeoeffective events.

(vi) The correlation between CME speed and flare flux is good for geoeffective events $(R=0.63)$ and weak for nongeoeffective events $(R=0.32)$.

(vii) The geoeffective events are found to be associated with intense geomagnetic storm with mean Dst index $(-150 \mathrm{nT})$. 
(viii) There exists a weak correlation between CME speed and Dst with a correlation coefficient of $R=-0.51$.

(ix) There exists a good correlation between (i) Dst index and solar wind speed with a correlation coefficient of $R=-0.64$, (ii) Dst index and southward magnetic field component $B_{z}$ with a correlation coefficient of $R=0.80$, and (iii) CME speed and solar wind speed with a correlation coefficient of $R=0.60$.

It is concluded that the intense and long duration southward magnetic field component $\left(B_{z}\right)$ and fast solar wind speed are responsible for geomagnetic storms; the geomagnetic storms weakly depend on CME speed since they are altered by solar wind in the interplanetary medium. The faster and wider CMEs are more energetic and drive shocks in the outer corona [11]. About 22\% of the DH-type-II bursts are associated with geomagnetic storms. Therefore the energetic CMEs that in turn produce the DH-type-II bursts are found to be associated with major flares. These DH-type-II bursts associated CMEs are good indicator of geomagnetic storms.

\section{Acknowledgment}

The authors greatly acknowledge the various online data centers of NOAA and NASA. They thank the Wind/WAVES team for providing the Type II catalog. The CME catalog used is provided by the center for solar physics and space weather and the catholic university of America in cooperation with Naval Research Laboratory and NASA. V. Vasanth thanks UGC-BSR for a meritorious fellowship.

\section{References}

[1] R. A. Howard, D. J. Michels, N. R. Sheeley, and M. J. Koomen, "The observation a coronal transient directed at earth," The Astrophysical Journal Letters, vol. 263, pp. L101-L104, 1982.

[2] B. T. Tsurutani, "The interplanetary cause of magnetic storms, sub storms and geomagnetic quiet," in Space Storm and Space Weather Hazard, I. A. Daglis, Ed., p. 101, Kluwer Academic Publishers, Dordrecht, The Netherlands, 2001.

[3] V. Bothmer and R. Schwenn, "The interplanetary and solar causes of major geomagnetic storms," Journal of Geomagnetism and Geoelectricity, vol. 47, pp. 1127-1132, 1995.

[4] J. T. Gosling, S. J. Bame, D. J. McComas, and J. L. Phillips, "Coronal mass ejections and large geomagnetic storms," Geophysical Research Letters, vol. 17, pp. 901-904, 1990.

[5] B. T. Tsurutani and W. D. Gonzalez, "The interplanetary cause of geomagnetic storms," in Magnetic Storms, B. T. Tsurutani, Ed., Geophysical Monograph Series, p. 77, AGU Press, Washington, DC, USA, 1998.

[6] G. E. Brueckner, J.-P. Delaboudiniere, R. A. Howard et al., "Geomagnetic storms caused by coronal mass ejections (CMEs): March 1996 through June 1997," Geophysical Research Letters, vol. 25, no. 15, pp. L3019-L3022, 1998.

[7] D. F. Webb, E. W. Cliver, N. U. Crooker, O. C. St. Cyr, and B. J. Thompson, "Relationship of halo coronal mass ejections, magnetic clouds, and magnetic storms," Journal of Geophysical Research A, vol. 105, no. 4, pp. 7491-7508, 2000.

[8] H. V. Cane, I. G. Richardson, and O. C. St. Cyr, "Coronal mass ejections, interplanetary ejecta and geomagnetic storms,"
Geophysical Research Letters, vol. 27, no. 21, pp. L3591-L3594, 2000.

[9] N. Gopalswamy, A. Lara, R. P. Lepping, M. L. Kaiser, D. Berdichevsky, and O. C. St. Cyr, "Interplanetary acceleration of coronal mass ejections," Geophysical Research Letters, vol. 27, no. 2, pp. 145-148, 2000.

[10] I. G. Richardson, E. W. Cliver, and H. V. Cane, "Sources of geomagnetic activity over the solar cycle: relative importance of coronal mass ejections, high-speed streams, and slow solar wind," Journal of Geophysical Research A, vol. 105, no. 8, pp. 18203-18213, 2000.

[11] N. Gopalswamy, S. Yashiro, M. L. Kaiser, R. A. Howard, and J.-L. Bougeret, "Characteristics of coronal mass ejections associated with long-wavelength type II radio bursts," Journal of Geophysical Research A, vol. 106, no. 12, pp. 29219-29229, 2001.

[12] N. Gopalswamy, M. L. Kaiser, B. J. Thompson et al., "Radio-rich solar eruptive events," Geophysical Research Letters, vol. 27, no. 10, pp. 1427-1430, 2000.

[13] J.-L. Bougeret, M. L. Kaiser, P. J. Kellogg et al., "WAVES: the radio and plasma wave investigation on the wind spacecraft," Space Science Reviews, vol. 71, no. 1-4, pp. 231-263, 1995.

[14] S. Yashiro, N. Gopalswamy, G. Michalek et al., "A catalog of white light coronal mass ejections observed by the SOHO spacecraft," Journal of Geophysical Research A, vol. 109, no. 7, p. 105, 2004.

[15] N. Gopalswamy, S. Yashiro, S. Akiyama et al., "Coronal mass ejections, type II radio bursts, and solar energetic particle events in the SOHO era," Annales Geophysicae, vol. 26, no. 10, pp. 30333047, 2008.

[16] I. G. Richardson and H. V. Cane, "Near-earth interplanetary coronal mass ejections during solar cycle 23 (1996-2009): catalog and summary of properties," Solar Physics, vol. 264, no. 1, pp. 189-237, 2010.

[17] N. Gopalswamy, S. Yashiro, and S. Akiyama, "Geoeffectiveness of halo coronal mass ejections," Journal of Geophysical Research $A$, vol. 112, no. 6, p. 112, 2007.

[18] N. Gopalswamy, E. Aguilar-Rodriguez, S. Yashiro, S. Nunes, M. L. Kaiser, and R. A. Howard, "Type II radio bursts and energetic solar eruptions," Journal of Geophysical Research A, vol. 110, no. 12, p. S07, 2005.

[19] N. Gopalswamy, "Coronal mass ejections and type II bursts," in Solar Eruptions and Energetic Particles, N. Gopalswamy, R. Mewaldt, and J. Torsti, Eds., vol. 165 of Geophysical Monograph Series, p. 207, The American Geophysical Union, 2006.

[20] V. Vasanth, S. Umapathy, B. Vršnak, and M. Anna Lakshmi, "Characteristics of type-II radio bursts associated with flares and CMEs," Solar Physics, vol. 273, no. 1, pp. 143-162, 2011.

[21] B. Vršnak, D. Ruždjak, D. Sudar, and N. Gopalswamy, "Kinematics of coronal mass ejections between 2 and 30 solar radii: what can be learned about forces governing the eruption?" Astronomy and Astrophysics, vol. 423, no. 2, pp. 717-728, 2004.

[22] B. Vrånak, "Processes and mechanisms governing the initiation and propagation of CMEs," Annales Geophysicae, vol. 26, no. 10, pp. 3089-3101, 2008.

[23] E. W. Cliver, D. F. Webb, and R. A. Howard, "On the origin of solar metric type II burst,” Solar Physics, vol. 187, no. 1, pp. 89114, 1999.

[24] B. Vršnak, "Deceleration of coronal mass ejections," Solar Physics, vol. 202, no. 1, pp. 173-189, 2001.

[25] J. T. Gosling, E. Hildner, R. M. MacQueen, R. H. Munro, A. I. Poland, and C. L. Ross, "The speeds of coronal mass ejection events," Solar Physics, vol. 48, no. 2, pp. 389-397, 1976. 
[26] Y.-J. Moon, G. S. Choe, H. Wang et al., "A statistical study of two classes of coronal mass ejections," The Astrophysical Journal Letters, vol. 581, no. 1, pp. 694-702, 2002.

[27] N. R. Sheeley Jr., J. H. Walters, Y.-M. Wang, and R. A. Howard, "Continuous tracking of coronal outflows: two kinds of coronal mass ejections," Journal of Geophysical Research A, vol. 104, no. 11, pp. 24739-24767, 1999.

[28] J. Zhang, K. P. Dere, R. A. Howard, and V. Bothmer, "Identification of solar sources of major geomagnetic storms between 1996 and 2000," The Astrophysical Journal Letters, vol. 582, no. 1, pp. 520-533, 2003.

[29] B. M. Bein, S. Berkebile-Stoiser, A. M. Veronig et al., "Impulsive acceleration of coronal mass ejections. I. Statistics and coronal mass ejection source region characteristics," The Astrophysical Journal, vol. 738, no. 2, article 191, pp. 1-14, 2011.

[30] P. J. Cargill and J. M. Schmidt, "Modelling interplanetary CMEs using magnetohydrodynamic simulations," Annales Geophysicae, vol. 20, no. 7, pp. 879-890, 2002.

[31] P. J. Cargill, J. Chen, D. S. Spicer, and S. T. Zalesak, "Magnetohydrodynamic simulations of the motion of magnetic flux tubes through a magnetized plasma," Journal of Geophysical Research, vol. 101, p. 4855, 1996.

[32] G. M. Lindsay, "Relationships between coronal mass ejection speeds from coronagraph images and interplanetary characteristics of associated interplanetary coronal mass ejections," Journal of Geophysical Research A, vol. 104, no. 6, pp. 1251512523, 1999.

[33] V. Vasanth and S. Umapathy, "A statistical study on CMEs associated with DH-type-II radio bursts based on their source location (limb and disk events)," Solar Physics, vol. 282, pp. 239247, 2013.

[34] N. Gopalswamy, A. Lara, S. Yashiro, M. L. Kaiser, and R. A. Howard, "Predicting the 1-AU arrival times of coronal mass ejections," Journal of Geophysical Research A, vol. 106, no. 12, pp. 29207-29217, 2001.

[35] N. Gopalswamy, A. Lara, P. K. Manoharan, and R. A. Howard, "An empirical model to predict the 1-AU arrival of interplanetary shocks," Advances in Space Research, vol. 36, no. 12, pp. 2289-2294, 2005.

[36] U. Bak-Steslicka, S. Kolomanski, and T. Mrozek, "Coronal mass ejections associated with slow long duration flares," Solar Physics, vol. 283, pp. 505-517, 2013.

[37] B. M. Bein, S. Berkebile-Stoiser, A. M. Veronig, M. Temmer, and B. Vrsnak, "Impulsive acceleration of coronal mass ejections. ii. Relation to soft $\mathrm{x}$-ray flares and filament eruptions," The Astrophysical Journal, vol. 755, no. 1, article 44, pp. 1-12, 2012.

[38] J. T. Burkepile, A. J. Hundhausen, A. L. Stanger, O. C. St. Cyr, and J. A. Seiden, "Role of projection effects on solar coronal mass ejection properties: 1 . A study of CMEs associated with limb activity," Journal of Geophysical Research A, vol. 109, no. 3, p. 103, 2004.

[39] D. Maričić, B. Vršnak, A. L. Stanger, A. M. Veronig, M. Temmer, and D. Roša, "Acceleration phase of coronal mass ejections: II. Synchronization of the energy release in the associated flare," Solar Physics, vol. 241, no. 1, pp. 99-112, 2007.

[40] Y.-J. Moon, G. S. Choe, H. Wang, Y. D. Park, and C. Z. Cheng, "Relationship between CME kinematics and flare strength," Journal of Korean Astronomical Society, vol. 36, pp. 61-66, 2003.

[41] S. Yashiro and N. Gopalswamy, "Statistical relationship between solar flares and coronal mass ejections," in Universal Heliophysical Processes, N. Gopalswamy and D. F. Webb, Eds., vol. 257 of IAU Symposium, pp. 233-243, International Astronomical Union, 2008.

[42] C. A. Loewe, "Classification and mean behavior of magnetic storms," Journal of Geophysical Research A, vol. 102, no. 7, pp. 14209-14213, 1997.

[43] N. Gopalswamy, "Coronal mass ejections and space weather," in Climate and Weather of the Sun-Earth System (CAWSES): Selected Papers from the 2007 Kyoto Symposium, T. Tsuda, R. Fujii, K. Shibata, and M. A. Geller, Eds., pp. 77-120, Terra Scientific Publishing, 2009. 

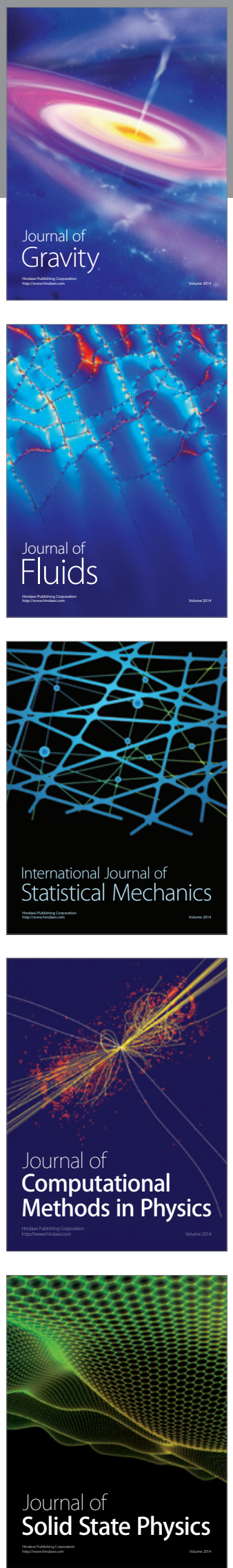

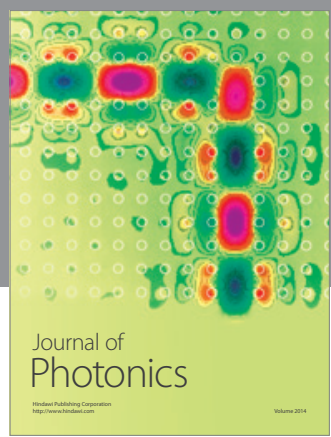

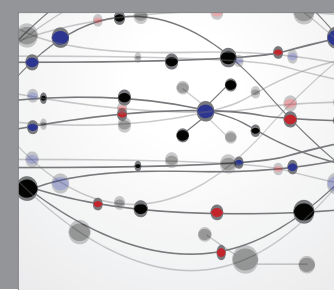

The Scientific World Journal

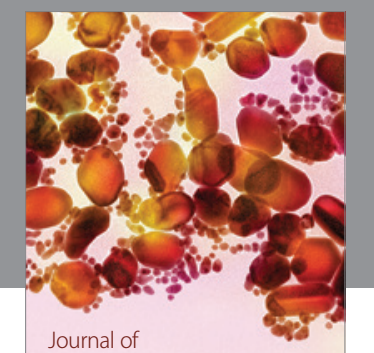

Soft Matter
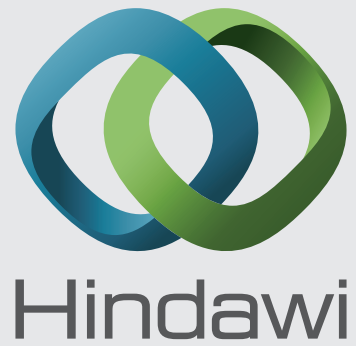

Submit your manuscripts at

http://www.hindawi.com
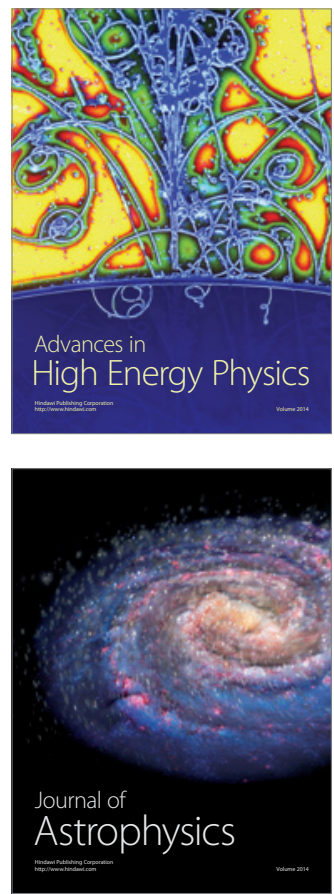
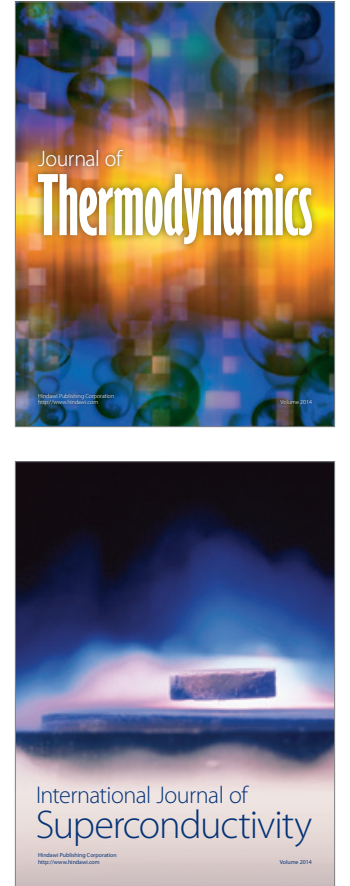
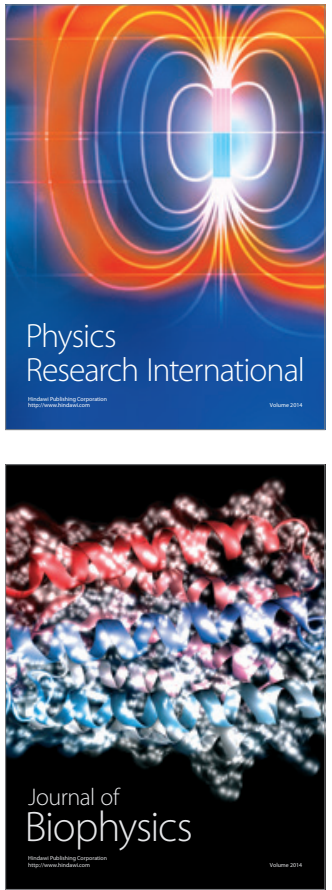
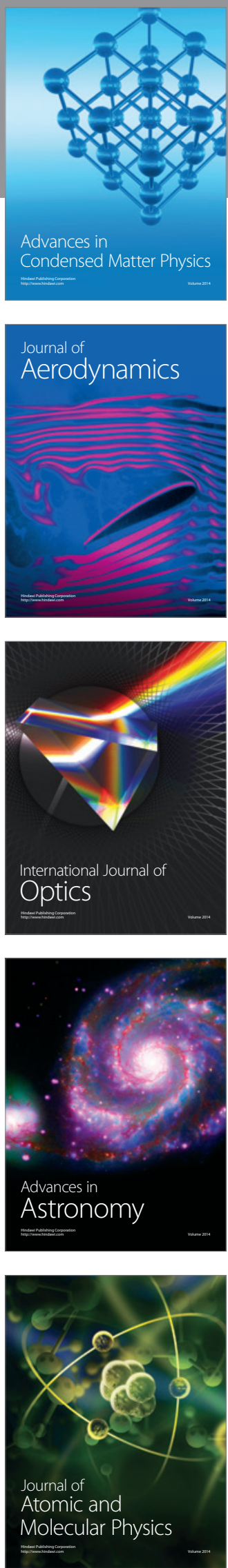